\title{
Correction to: Introduction to Digital Communications
}

\section{Correction to: \\ J. Speidel, Introduction to Digital Communications, Signals and Communication Technology, https://doi.org/10.1007/978-3-030-00548-1}

The book chapters $7,8,12,17$ and 23 of the original version were revised. The erratum chapter and the book have been updated with these changes.

The updated version of these chapters can be found at https://doi.org/10.1007/978-3-030-00548-1_7 https://doi.org/10.1007/978-3-030-00548-1_8 https://doi.org/10.1007/978-3-030-00548-1_12 https://doi.org/10.1007/978-3-030-00548-1_17 https://doi.org/10.1007/978-3-030-00548-1_23 